\title{
TAI CHI CHUAN MELHORA FUNCIONALIDADE E QUALIDADE DE VIDA EM HOMENS IDOSOS COM BAIXA DENSIDADE MINERAL ÓSSEA
}

\author{
Tai Chi Chuan improves functionality and quality \\ of life in elderly men with low bone mineral density
}

\author{
Márcio Moura Pereiraa, Vinicius Carolino Souza ${ }^{b}$, Ana Patrícia Paulab, \\ Clayton Franco Moraesa, Otávio Toledo Nóbregaba Lucy Gomes ${ }^{\mathrm{a}}$
}

OBJETIVO: Avaliar efeitos do Tai Chi Chuan (TCC) na capacidade funcional (CF) e na qualidade de vida (QV) de homens idosos com baixa densidade mineral óssea (DMO). MÉTODO: Estudo quasi-experimental, controlado, cego, com 41 idosos ( $\geq 60$ anos) divididos em dois grupos: grupo experimental - G1 (TCC; $n=20 ; 69,2 \pm 6,2$ anos) e grupo controle $-G 2$ (controle; $n=21$; 69,0 \pm 5,7 anos). A DMO (coluna lombar e colo do fêmur) foi avaliada com raios-x de dupla energia (DEXA). Para aferir CF foram utilizados: testes de resistência aeróbia (RA); de força e flexibilidade de membros superiores e inferiores; de equilíbrio em condições estática, dinâmica e funcional; e de alcance funcional, assim como para risco de quedas. A QV foi pesquisada pelo questionário SF-36 (MOS 36 ltem Short-Form Health Survey). O G1 praticou TCC estilo Yang, com 24 movimentos por 12 semanas, 2 vezes por semana, em intensidade leve; o G2 não praticou atividade física orientada. RESULTADOS: Praticantes de TCC apresentaram valores significativamente maiores em resistência aeróbia, em força de membros superiores e inferiores, e para equilíbrio dinâmico. Ao aferir a QV, surgiu significância para escore total, assim como para as dimensões de capacidade funcional, estado geral de saúde, vitalidade e saúde mental. DISCUSSÃO: Constataram-se efeitos benéficos do TCC em CF e QV, o que agrega evidências à sua utilização em homens idosos com baixa DMO. CONCLUSÃO: O TCC é eficaz na melhora da CF e da QV em homens idosos com baixa DMO.

PALAVRAS-CHAVE: tai chi chuan; idoso; osteoporose; qualidade de vida.

OBJECTIVE: The purpose was to assess the effect of Tai Chi Chuan (TCC) on functional capacity (FC) and quality of life (QoL) in elderly men with low bone mineral density (BMD). METHODS: This quasi-experimental, controlled blinded trial evaluated 41 senior men ( $\geq 60$ years), divided into two groups: control group 1 - G1 (TCC; $n=20 ; 69.2 \pm 6.2$ years) and control group 2 - G2 (control; $\mathrm{n}=21 ; 69.0 \pm 5.7$ years). The BMD (of lumbar spine and femur neck) was assessed by dual energy $x$-ray absorptiometry (DEXA).

$\checkmark \quad F C$ was assessed for aerobic endurance, for upper and lower limbs strength and flexibility, for static, dynamic and functional $\varangle \quad$ balance testes, and for risk of falls. QoL was assessed by the SF-36 questionnaire (MOS 36-item Short-Form Health Survey). is G1 practiced the 24-form Yang style of TCC, for 12 weeks twice a week on low intensity; G2 did not practice oriented physical activity. RESULTS: TCC practitioners had significantly higher scores for aerobic endurance, upper and lower limbs strength, and dynamic balance. In terms of QoL, values were significantly better in total score and aspects such as FC, general health, vitality, and mental health. DISCUSSION: TCC was proven beneficial to FC and QoL scores, which adds evidence for its practice by elderly men with low BMD. CONCLUSION: TCC training is effective in improving FC and QoL in elderly men with low BMD. KEYWORDS: tai chi chuan; aged; osteoporosis; quality of life. 


\section{INTRODUÇÃO}

A osteoporose é uma doença esquelética sistêmica caracterizada por baixa densidade mineral óssea (DMO) e comprometimento da resistência óssea, levando à fragilidade dos ossos e ao consequente aumento do risco de fraturas. No Brasil, a doença foi autorreferida por 5,1\% dos homens idosos. ${ }^{1}$ Estudo realizado com 151 homens no município do Rio de Janeiro revelou prevalência progressiva da osteoporose conforme o aumento da idade - nos indivíduos com idade entre 60 e 69 anos foi constatada uma prevalência de 16,2\%; entre 70 e 79 anos, 34,2\%; e a partir de 80 anos, 48,6\%. ${ }^{2}$

Embora a osteoporose seja descrita mais frequentemente em mulheres, dados epidemiológicos sugerem que o risco de fratura, principalmente espinhal, é maior nos homens, sendo a mortalidade decorrente superior à de mulheres nas mesmas condições. ${ }^{3}$ Como consequência das fraturas e suas sequelas, os indivíduos perdem sua capacidade funcional (CF). Muitos deles, devido às fraturas vertebrais, apresentam aumento da cifose torácica e alterações do equilíbrio. Homens idosos com osteoporose apresentam comprimento da passada encurtada, menor flexibilidade de quadril e de membros inferiores, assim como menor velocidade da marcha. ${ }^{4}$

Exercícios resistidos são os recomendáveis para pacientes com osteoporose, pois melhoram a força muscular, a flexibilidade e a coordenação, assim como promovem elevação em DMO e diminuição de risco de quedas. ${ }^{5}$ Entretanto, programas de saúde pública que utilizam esse tipo de prática apresentam limitações para inserção em grandes populações, pois geralmente exigem atendimento individualizado, além de espaço físico e equipamentos específicos. Assim, exercícios com sobrecarga figuram como coadjuvantes do tratamento medicamentoso, enquanto os de equilíbrio e de coordenação são essenciais, tendo função independente na prevenção de quedas e de fraturas traumáticas.

Nesse contexto, o Tai Chi Chuan (TCC) consiste em um exercício apropriado para sujeitos idosos: pode ser prescrito com intensidades variadas - leve a intensa -, não requer equipamentos específicos e proporciona efeitos benéficos à saúde devido ao seu caráter não estressor e não competitivo. $\mathrm{O}$ exercício tem ainda a vantagem de apresentar baixo custo, fácil aplicabilidade e potencial para atender a grandes grupos, com flexibilidade quanto ao local, horário e roupas para sua prática. Constitui atividade física que atende às recomendações do American College of Sports Medicine para incremento da CF, com seu principal efeito no equilíbrio e na redução de quedas, ${ }^{6}$ melhorando também a capacidade aeróbia, a força muscular e o balanço. ${ }^{7}$

Há evidências de que a prática regular do TCC promove ganhos na qualidade de vida (QV), incluindo bem-estar emocional (particularmente humor, estresse, ansiedade, depressão e tensão produzida por raiva), autopercepção (autoeficácia e medo de cair) e bem-estar físico (dor crônica e desordens do sono). ${ }^{8,9}$

No Brasil, em programas governamentais de saúde pública, não há acordo sobre quais são os exercícios adequados para sujeitos portadores de baixa DMO que podem ser utilizados amplamente na comunidade. Dessa forma, o objetivo deste estudo foi verificar os efeitos de um programa de TCC sobre a $\mathrm{CF}$ e a $\mathrm{QV}$ de homens idosos com baixa DMO vivendo em uma comunidade.

\section{MÉTODOS}

\section{Sujeitos e intervenção}

Foi realizado estudo quasi-experimental, com delineamento controlado e cego para os avaliadores, em amostra de conveniência, composta de homens idosos (idade $\geq 60$ anos) cadastrados no Programa de Prevenção e Diagnóstico da Osteoporose da Secretaria de Saúde do Distrito Federal (DF). Os sujeitos eram atendidos em unidade de saúde da rede pública pela mesma equipe de profissionais de saúde. O estudo foi aprovado pelo Comitê de Ética em Pesquisa da Universidade de Brasília (CEP/UnB), sob o protocolo $n^{\circ} 145 / 2008$.

Além do sexo e da faixa etária, figuraram como critérios de inclusão na amostra:

- ser portador de baixa DMO (T-score $\leq 1)$, com diagnóstico clínico e densitométrico de osteopenia ou osteoporose;

- não ser praticante regular de atividade física orientada nos últimos três meses;

- ter indicação clínica para a prática de exercícios físicos; e

- ter assinado o termo de consentimento livre e esclarecido (TCLE).

Os critérios de exclusão consistiram em apresentar doenças ou limitações incapacitantes à realização em testes de CF (tendinite; tenossinovite; osteoartrite sintomática; síndromes dolorosas, como fibromialgia e lombalgias; e síndromes neurológicas, como labirintite e enxaqueca), déficit cognitivo, verificado com o miniexame do estado mental de Folstein, que impedisse o entendimento das instruções de segurança para realização dos testes motores; diabetes mellitus e outras endocrinopatias.

Dos 297 homens idosos cadastrados no Programa de Prevenção e Diagnóstico na Unidade de Saúde investigada, 78 aceitaram participar do estudo, dos quais 41 preencheram 
os critérios de inclusão. Entre os 37 idosos excluídos, 21 apresentaram DMO normal, enquanto os 16 que mostraram baixa DMO foram excluídos por exibirem comorbidades como osteoartrite, lombalgia, hipertireoidismo e diabetes.

A amostra foi distribuída, por conveniência, em grupo experimental (G1), submetido à prática do TCC, e grupo controle (G2), sem prática de atividade física orientada no mesmo período. Os dois grupos mantiveram inalteradas suas atividades da vida diária (AVDs) durante o tempo do estudo. Foram realizadas avaliações em dois momentos $(\mathrm{T} 1=$ préteste e T2 = pós-teste) para as variáveis dependentes $\mathrm{CF}$ e $\mathrm{QV}$.

Os testes foram aplicados pelo primeiro autor da pesquisa em conjunto com acadêmicos de fisioterapia adequadamente treinados e sob supervisão durante a execução dos testes. Nenhum dos avaliadores tinha conhecimento sobre o grupo no qual o paciente estava incluído.

As aulas de TCC ocorreram no período matutino, em espaço aberto, nivelado e sombreado, sendo ministradas por profissional de educação física experiente. Os exercícios foram planejados com base no estilo Yang de 24 movimentos adaptados para idosos, levando-se em consideração a segurança dos pacientes. Utilizaram-se exercícios com grau baixo de dificuldade, coreografias curtas e poucas mudanças de direção. A intensidade do exercício foi leve, com percepção subjetiva do esforço (PSE) de 2 a 3 na escala de Borg para o esforço percebido. O programa de TCC teve duração de 12 semanas, com aulas de 50 minutos em duas sessões semanais. As práticas obedeceram às três partes recomendadas em aulas de educação física: aquecimento, treinamento e relaxamento. $\mathrm{O}$ aquecimento (15 minutos) foi composto por exercícios educativos, selecionados entre os 24 movimentos de treinamento do TCC, sendo executados com ênfase no alongamento muscular e nos exercícios respiratórios. No treinamento específico (20 minutos), os idosos executaram as coreografias peculiares do TCC, mantendo as características de lentidão, fluidez e concentração mental. Essas coreografias foram constituídas de 8 movimentos escolhidos da série de 24 movimentos, sendo também utilizadas as em dupla (Tai Chi Tuishou) baseadas nos 24 movimentos da série padrão. No relaxamento (15 minutos), buscou-se a descontração psíquica e muscular por meio de exercícios de meditação dinâmica (Tao Yin), que utilizam os treinos da série do TCC e os sentados (sentar na calma), a fim de produzir relaxamento muscular, recuperar o ritmo natural da respiração e desenvolver concentração mental.

\section{Índice de massa corporal}

A massa corporal $(\mathrm{kg})$ foi mensurada utilizando-se balança digital, com resolução de 0,01 kg e carga máxima de $150 \mathrm{~kg}$ (Toledo, SP, Brasil). Para a estatura (m), utilizouse estadiômetro, com resolução de $1 \mathrm{~mm}$ e estatura máxima de $2 \mathrm{~m}$ (Cardiomed, PR, Brasil). Foi calculado o índice de massa corporal (IMC).

\section{Densitometria mineral óssea}

Aferida nos sítios L2-L4 da coluna lombar e no colo do fêmur por absortometria com raio-x de dupla energia (DEXA), em aparelho Lunar modelo DPX-NT (GE Healthcare, UK). A técnica foi executada por avaliador treinado e experiente, seguindo as normas do fabricante, incluindo controle de qualidade e regras de calibração diária do equipamento. Os valores registrados da densitometria mineral óssea foram expressos em valores absolutos $\left(\mathrm{g} / \mathrm{cm}^{2}\right)$ e em T-scores comparativos com a população jovem saudável. Foram considerados normais os homens que apresentaram $\mathrm{T}$-score $>-1$ nas duas regiões avaliadas; e como portadores de baixa DMO (osteopenia ou osteoporose) aqueles com $\mathrm{T}$-score $\leq-1 \mathrm{em}$ pelo menos uma das regiões avaliadas. ${ }^{10}$

\section{Capacidade funcional}

Para avaliação da CF, foi utilizada a bateria Fullerton (Functional Fitness Test) desenvolvida por Rikli \& Jones, ${ }^{11}$ constando dos seguintes testes:

1. Teste 2-minute walk (teste da marcha estacionária de dois minutos - TME2'), na avaliação da resistência aeróbia (RA);

2. Teste 30-Second Chair Stand (sentar e levantar da cadeira em 30 segundos), verificando força de membros inferiores (FMMII);

3. Teste Back Scratch, medindo flexibilidade de membros superiores (FLEXMMSS);

4. Teste Chair Sit-and-Reach (sentar e alcançar), analisando flexibilidade de membros inferiores (FLEXMMII); $\mathrm{e}$

5. Teste 8-Foot Timed-Up-and-Go (8F-TUG), medindo o equilíbrio dinâmico.

O teste de caminhada de seis minutos foi substituído pelo TME2', porque ambos são capazes de avaliar a RA do mesmo modo, e por ser o segundo mais rápido e não necessitar de espaço físico amplo para sua realização.

Foi mensurada a força de membros superiores (FMMSS) por preensão manual isométrica (kg.f) em dinamômetro da marca Jamar (Asimow Engineering Co., CA, EUA), com capacidade para $90 \mathrm{~kg}$.f e resolução de $1 \mathrm{~kg} . f$.

Avaliou-se ainda o equilíbrio estático pelo teste de apoio unipodal com restrição visual (UNIPODAL); o controle postural anterior pelo teste de alcance funcional (TAF); o equilíbrio corporal (dinâmico e estático) com uso da escala de 
equilíbrio de Berg (EEB) adaptada para idosos brasileiros; e o índice de possibilidade de queda (IPQ), calculado a partir dos escores obtidos na EEB combinados com informações sobre o histórico de quedas após os 60 anos.

\section{Qualidade de vida}

A QV foi avaliada com o questionário curto relacionado à saúde com 36 questões (MOS 36 ltem Short-Form Health Survey-SF-36), traduzido para a língua portuguesa e validado no Brasil.

\section{Análise estatística}

Para análise descritiva dos dados, foram utilizados média $(\bar{X})$ e desvio padrão (DP). A hipótese de que, antes da intervenção, a distribuição dos diagnósticos de osteopenia e osteoporose seria diferente entre $\mathrm{G} 1$ e $\mathrm{G} 2$ foi experimentada por meio do teste do $\chi^{2}$. Os testes de Shapiro-Wilk e de Levene foram usados para avaliar, respectivamente, normalidade e homocedasticidade. Quando as variáveis não apresentaram distribuições normais e homocedasticidade, utilizou-se na comparação dos grupos o teste $t$ de Student para medidas independentes e o teste de Mann-Whitney para variáveis contínuas e categóricas. Análises estatísticas inferenciais foram aplicadas para verificar os efeitos entre e intragrupos. Para comparação dos dados paramétricos, foi utilizada a análise de variância (Splitplot Anova) com testes post-hoc de least-significance difference (LSD), e, no confronto dos dados não paramétricos, foram empregados os testes do $\chi^{2}$, Mann-Whitney e Wilcoxon com correção de Bonferroni. O nível de significância utilizado foi $\mathrm{p} \leq 0,05$. Os procedimentos estatísticos foram executados no programa SPSS (Statistical Package for the Social Sciences) para Windows 18.0 (IBM, New York, EUA).

\section{RESULTADOS}

Não houve perda amostral no período do estudo. Os 41 homens idosos estudados com baixa DMO foram distribuídos entre o G1 e o G2 - 20 e 21 indivíduos, respectivamente. As medidas descritivas por idade, antropometria e densitometria estão apresentadas na Tabela 1.

Os grupos $\mathrm{G} 1$ e G2 apresentaram distribuições equiparáveis $(\mathrm{p}>0,05)$ em relação à média e à frequência para a maioria de suas medidas (sexo, idade, DMO, equilíbrio corporal, capacidade aeróbia, capacidade cognitiva, escolaridade e estado marital). Verificou-se não haver diferenças significativas entre as variáveis no início do experimento, descartando-se, portanto, a hipótese de que, antes da intervenção, a distribuição dos diagnósticos de osteopenia e osteoporose seria diferente entre os grupos $\left(\chi^{2}=1,18 ; p=0,35\right)$. Assim, verificou-se que, embora os participantes dos grupos G1 e G2 tenham sido distribuídos por conveniência, de forma não aleatória, mostraram-se semelhantes nas variáveis descritivas obtidas no início do experimento, garantindo que o número de indivíduos com os dois diagnósticos em cada grupo fosse semelhante.

\section{Efeito do tai chi chuan sobre a capacidade funcional}

Os resultados das comparações das medianas obtidas para $\mathrm{CF}$ entre e intragrupos, após o treinamento, estão expostos na Tabela 2.

Verificou-se que a prática do TCC promoveu melhora significativa $(p=0,02)$ na CF avaliada na resistência aeróbia (F[1$39]=6,08 ; \mathrm{p}=0,02)$, assim como na FMMII (F[1-39] = 9,49; $\mathrm{p}=0,01)$ e na FMMSS $(\mathrm{F}[1-39]=4,34 ; \mathrm{p}=0,04)$. Ao final do treinamento, os praticantes de TCC exibiram desempenho significativamente melhor de membros inferiores, tanto na comparação entre grupos $(\mathrm{p}=0,03)$ quanto na intragrupos $(\mathrm{p}=0,01)$. No tocante à flexibilidade, a prática do TCC não promoveu alterações na FLEXMMSS e na FLEXMMII no $\mathrm{G} 1$ em relação ao $\mathrm{G} 2$.

Tabela 1 Caracterização dos 41 homens idosos com baixa densidade mineral óssea nos grupos experimental e controle.

\begin{tabular}{|c|c|c|c|}
\hline Variáveis & Grupos & $\bar{X} \pm D P$ & Valor $p$ \\
\hline \multirow{2}{*}{ Idade (anos) } & G1 & $69,2 \pm 6,2$ & \multirow{2}{*}{0,91} \\
\hline & $\mathrm{G} 2$ & $69,0 \pm 5,7$ & \\
\hline \multicolumn{4}{|l|}{ Antropometria } \\
\hline \multirow{2}{*}{ Massa (kg) } & G1 & $67,7 \pm 9,4$ & \multirow{2}{*}{0,67} \\
\hline & $\mathrm{G} 2$ & $69,6 \pm 11,4$ & \\
\hline \multirow{2}{*}{ Estatura (cm) } & $\mathrm{G} 1$ & $166,0 \pm 6,0$ & \multirow{2}{*}{0,42} \\
\hline & $\mathrm{G} 2$ & $168,0 \pm 5,0$ & \\
\hline \multirow{2}{*}{ IMC $\left(\mathrm{kg} / \mathrm{m}^{2}\right)$} & $\mathrm{G} 1$ & $24,5 \pm 3,1$ & \multirow{2}{*}{0,63} \\
\hline & $\mathrm{G} 2$ & $24,6 \pm 3,8$ & \\
\hline \multicolumn{4}{|l|}{ Densitometria } \\
\hline \multirow{2}{*}{ DMO fêmur (g/cm²) } & G1 & $0,84 \pm 0,08$ & \multirow{2}{*}{0,99} \\
\hline & $\mathrm{G} 2$ & $0,85 \pm 0,11$ & \\
\hline \multirow{2}{*}{ T-score fêmur } & G1 & $-1,79 \pm 0,65$ & \multirow{2}{*}{0,92} \\
\hline & $\mathrm{G} 2$ & $-1,68 \pm 0,89$ & \\
\hline \multirow{2}{*}{ DMO lombar $\left(\mathrm{g} / \mathrm{cm}^{2}\right)$} & $\mathrm{G} 1$ & $0,94 \pm 0,08$ & \multirow{2}{*}{0,46} \\
\hline & G2 & $0,96 \pm 0,15$ & \\
\hline \multirow{2}{*}{ T-score lombar } & $\mathrm{G} 1$ & $-2,29 \pm 0,71$ & \multirow{2}{*}{0,40} \\
\hline & $\mathrm{G} 2$ & $-2,20 \pm 1,26$ & \\
\hline
\end{tabular}

X: média; DP: desvio padrão; p: significância na comparação entre grupos; G1: grupo experimental; G2: grupo controle; IMC: índice de massa corporal; DMO: densidade mineral óssea. 
Nas comparações do equilíbrio, a prática do TCC promoveu alterações significativas apenas no equilíbrio dinâmico medido no teste 8F-TUG (F[1-39] = 10,82; $\mathrm{p}=0,01)$. No teste de equilíbrio de Berg não ocorreram diferenças significativas. Ao final do treinamento do TCC, houve redução significativa do tempo médio de realização desse teste, tanto na comparação entre grupos $(\mathrm{p}=0,02)$ quanto na intragrupos $(\mathrm{p}=0,001)$.

Tabela 2 Capacidade funcional nos 41 homens idosos com baixa densidade mineral óssea.

\begin{tabular}{|c|c|c|c|c|}
\hline Testes & Grupos & $\begin{array}{l}\text { Pré-treino } \\
(\bar{X} \pm D P)\end{array}$ & $\begin{array}{c}\text { Pós-treino } \\
(\bar{X} \pm \text { DP) }\end{array}$ & $\begin{array}{c}\text { Valor } \\
\mathbf{p}\end{array}$ \\
\hline \multicolumn{5}{|c|}{ Resistência aeróbia } \\
\hline \multirow{2}{*}{ RA (passos) } & G1 & $93,3 \pm 11,8$ & $101,7 \pm 20,8^{*}$ & \multirow{2}{*}{0,02} \\
\hline & $\mathrm{G} 2$ & $92,5 \pm 10,4$ & $88,7 \pm 12,8$ & \\
\hline \multicolumn{5}{|l|}{ Força muscular } \\
\hline \multirow{2}{*}{$\begin{array}{l}\text { FMMII } \\
\text { (levantamentos) }\end{array}$} & G1 & $15,5 \pm 1,3$ & $18,3 \pm 4,7^{*}$ & \multirow{2}{*}{0,01} \\
\hline & $\mathrm{G} 2$ & $14,7 \pm 3,2$ & $15,3 \pm 3,0$ & \\
\hline \multirow{2}{*}{ FMMSS (kg.f) } & G1 & $36,5 \pm 6,4$ & $39,0 \pm 11,3^{*}$ & \multirow{2}{*}{0,04} \\
\hline & $\mathrm{G} 2$ & $33,2 \pm 7,8$ & $32,4 \pm 6,6$ & \\
\hline \multicolumn{5}{|l|}{ Flexibilidade } \\
\hline \multirow{2}{*}{$\begin{array}{l}\text { FLEXMMII } \\
(\mathrm{cm})\end{array}$} & G1 & $16,2 \pm 9,4$ & $17,8 \pm 7,5$ & \multirow{2}{*}{0,25} \\
\hline & $\mathrm{G} 2$ & $18,9 \pm 11,0$ & $21,8 \pm 10,2$ & \\
\hline \multirow{2}{*}{$\begin{array}{l}\text { FLEXMMSS } \\
(\mathrm{cm})\end{array}$} & G1 & $-11,3 \pm 7,9$ & $-11,5 \pm 8,1$ & \multirow{2}{*}{0,44} \\
\hline & $\mathrm{G} 2$ & $-13,0 \pm 9,8$ & $-14,6 \pm 10,8$ & \\
\hline \multicolumn{5}{|l|}{ Equilíbrio } \\
\hline \multirow{2}{*}{ UNIPODAL (s) } & G1 & $3,0 \pm 0,9$ & $3,0 \pm 1,5$ & \multirow{2}{*}{0,27} \\
\hline & $\mathrm{G} 2$ & $2,7 \pm 1,4$ & $3,2 \pm 1,6$ & \\
\hline \multirow{2}{*}{ 8F-TUG (s) } & G1 & $5,9 \pm 0,9$ & $4,8 \pm 0,5^{*}$ & \multirow{2}{*}{0,01} \\
\hline & $\mathrm{G} 2$ & $6,1 \pm 1,2$ & $5,4 \pm 1,2$ & \\
\hline \multirow{2}{*}{ TAF (cm) } & G1 & $35,4 \pm 7,4$ & $34,6 \pm 9,1$ & \multirow{2}{*}{0,87} \\
\hline & $\mathrm{G} 2$ & $36,5 \pm 6,6$ & $35,1 \pm 6,2$ & \\
\hline \multirow{2}{*}{ EEB (escore) } & G1 & $54,1 \pm 4,0$ & $55,3 \pm 0,9$ & \multirow{2}{*}{0,32} \\
\hline & $\mathrm{G} 2$ & $53,0 \pm 5,8$ & $54,4 \pm 2,3$ & \\
\hline \multirow{2}{*}{ IPQ (\%) } & G1 & $9,5 \pm 23,1$ & $2,7 \pm 4,6$ & \multirow{2}{*}{0,20} \\
\hline & $\mathrm{G} 2$ & $17,9 \pm 34,2$ & $9,5 \pm 21,1$ & \\
\hline
\end{tabular}

$\bar{X}$ : média; DP: desvio padrão; $p$ : significância ( $x \leq 0,05)$; $G 1$ : grupo experimental; G2: grupo controle; RA: resistência aeróbia (teste da marcha estacionária de dois minutos); FMMII: força de membros inferiores (sentar e levantar da cadeira); FMMSS: força de membros superiores (preensão manual); FLEXMMII: flexibilidade de membros inferiores (sentar e alcançar); FLEXMMSS: flexibilidade de membros superiores (alcançar atrás das costas); UNIPODAL: apoio unipodal (com restrição visual); 8F-TUG: Eight-Foot Timed-Up-and-Go (sentado, caminhar e voltar a sentar); TAF: teste de alcance funcional; EEB: escala de equilíbrio de Berg; IPQ: índice de possibilidade de queda; *mensuração com diferença significativa nos testes post-hoc.
Nos dois tempos medidos, não surgiram diferenças significativas entre os grupos no equilíbrio estático (UNIPODAL), no TAF, na EEB e no IPQ.

\section{Efeito do TCC sobre a QV}

Não foram encontradas diferenças significativas entre os escores do SF-36, comparando-se os valores medianos dos dois grupos no início do experimento. Os resultados das comparações das medianas entre e intragrupos na QV total e em seus domínios são apresentados na Tabela 3.

A prática do TCC promoveu alterações significativas no escore total da QV $(z=-2,11 ; p=0,04)$. Quanto aos oito domínios compreendidos no teste, os relacionados à $\mathrm{CF}$ $(\mathrm{z}=-2,51 ; \mathrm{p}=0,01)$, ao estado geral de saúde $(\mathrm{z}=-2,42$; $\mathrm{p}=0,02)$, à vitalidade $(\mathrm{z}=-2,38 ; \mathrm{p}=0,02)$ e à saúde mental $(z=-2,34 ; p=0,02)$ sofreram alterações significativas após a prática dessa atividade.

Não houve diferença significativa entre grupos na comparação entre os domínios relativos à dor e aos aspectos físico, social e emocional.

Tabela 3 Medidas de qualidade de vida em 41 homens idosos com baixa densidade mineral óssea.

\begin{tabular}{|c|c|c|c|c|}
\hline $\begin{array}{l}\text { Domínios } \\
\text { da QV }\end{array}$ & Grupos & $\begin{array}{l}\text { Pré-treino } \\
(\bar{X} \pm D P)\end{array}$ & $\begin{array}{l}\text { Pós-treino } \\
(\bar{x} \pm D P)\end{array}$ & $\begin{array}{c}\text { Valor } \\
\text { p }\end{array}$ \\
\hline \multirow{2}{*}{$\begin{array}{l}\text { QV1 } \\
\text { Capacidade } \\
\text { funcional }\end{array}$} & G1 & $79,2 \pm 17,3$ & $90,8 \pm 10,6^{*}$ & \multirow{2}{*}{0,01} \\
\hline & $\mathrm{G} 2$ & $80,2 \pm 19,8$ & $72,4 \pm 26,4$ & \\
\hline \multirow{2}{*}{$\begin{array}{l}\text { QV2 Aspecto } \\
\text { físico }\end{array}$} & G1 & $95,0 \pm 13,1$ & $86,2 \pm 26,2$ & \multirow{2}{*}{0,52} \\
\hline & $\mathrm{G} 2$ & $86,9 \pm 18,7$ & $79,8 \pm 30,2$ & \\
\hline \multirow{2}{*}{ QV3 Dor } & $\mathrm{G} 1$ & $62,6 \pm 23,3$ & $71,8 \pm 25,6$ & \multirow{2}{*}{0,13} \\
\hline & $\mathrm{G} 2$ & $72,4 \pm 29,2$ & $60,2 \pm 25,4$ & \\
\hline \multirow{2}{*}{$\begin{array}{l}\text { QV4 Estado } \\
\text { geral de saúde }\end{array}$} & $\mathrm{G} 1$ & $78,3 \pm 15,9$ & $88,2 \pm 15,1^{*}$ & \multirow{2}{*}{0,02} \\
\hline & $\mathrm{G} 2$ & $82,0 \pm 10,0$ & $77,2 \pm 17,8$ & \\
\hline \multirow{2}{*}{ QV5 Vitalidade } & $\mathrm{G} 1$ & $76,5 \pm 17,2$ & $84,8 \pm 19,8^{*}$ & \multirow{2}{*}{0,02} \\
\hline & $\mathrm{G} 2$ & $76,4 \pm 15,9$ & $73,6 \pm 22,2$ & \\
\hline \multirow{2}{*}{$\begin{array}{l}\text { QV6 Aspectos } \\
\text { sociais }\end{array}$} & $\mathrm{G} 1$ & $84,4 \pm 22,8$ & $91,4 \pm 15,7$ & \multirow{2}{*}{0,29} \\
\hline & $\mathrm{G} 2$ & $88,1 \pm 21,4$ & $81,1 \pm 27,7$ & \\
\hline \multirow{2}{*}{$\begin{array}{l}\text { QV7 Aspecto } \\
\text { emocional }\end{array}$} & $\mathrm{G} 1$ & $98,4 \pm 7,2$ & $96,7 \pm 10,2$ & \multirow{2}{*}{0,33} \\
\hline & $\mathrm{G} 2$ & $87,3 \pm 24,8$ & $84,1 \pm 34,4$ & \\
\hline \multirow{2}{*}{$\begin{array}{l}\text { QV8 Saúde } \\
\text { mental }\end{array}$} & G1 & $82,4 \pm 15,6$ & $89,8 \pm 11,1^{*}$ & \multirow{2}{*}{0,02} \\
\hline & $\mathrm{G} 2$ & $86,5 \pm 12,2$ & $76,6 \pm 20,6$ & \\
\hline \multirow{2}{*}{ QV Escore total } & G1 & $82,1 \pm 10,7$ & $87,4 \pm 12,1^{*}$ & \multirow{2}{*}{0,04} \\
\hline & $\mathrm{G} 2$ & $82,5 \pm 13,8$ & $75,6 \pm 20,4$ & \\
\hline
\end{tabular}

QV: qualidade de vida; $\bar{X}$ : média; DP: desvio padrão; $p$ : significância ( $p \leq 0,05)$; G1: grupo experimental; G2: grupo controle; *mensuração com diferença significativa. 


\section{DISCUSSÃO}

\section{Capacidade funcional}

A avaliação dos efeitos do TCC sobre as variáveis representativas da $\mathrm{CF}$ confirmou achados descritos anteriormente, que relataram benefícios dessa atividade sobre diferentes parâmetros da CF. ${ }^{12} \mathrm{Na}$ pesquisa atual, a melhora observada na CF (RA, FMMII, FMMSS e equilíbrio dinâmico) acrescenta evidências em favor da utilização do TCC no condicionamento físico e na funcionalidade de idosos, ampliando esse benefício aos homens portadores de baixa DMO.

No presente trabalho, foram empregados cinco testes diferentes na avaliação do equilibrio, à semelhança do estudo de Voukelatos et al. ${ }^{13}$, que aplicaram uma bateria de seis testes visando diferentes aspectos dessa variável. Esses autores constataram benefícios significativos do TCC (praticado no estilo Sun, uma hora por semana) sobre o desempenho dos praticantes em todos os testes - com exceção do TAF — assim como a redução no número de quedas. Este estudo encontrou melhora somente no equilíbrio dinâmico no teste 8F-TUG, não havendo diminuição no risco de quedas. Desse modo, os dois estudos são concordantes quanto ao benefício do TCC sobre o equilíbrio, em algum grau, mas divergem quanto à abrangência desses efeitos. Uma possível explicação para esses achados discordantes seria os diferentes estilos de TCC utilizados nessas intervenções. Ademais, os autores citados trabalharam uma amostra heterogênea quanto ao sexo ( $85 \%$ eram mulheres).

Li et al. ${ }^{14}$ avaliaram o equilíbrio estático e dinâmico, assim como a flexibilidade em idosos, utilizando o mesmo estilo de TCC de nossa investigação (estilo Yang de 24 movimentos), porém com um protocolo ligeiramente diferente (menor frequência semanal, duração de um ano). Esses autores não verificaram melhora significativa nas variáveis estudadas no grupo praticante de TCC. Entretanto, Yang et al. ${ }^{15}$ utilizaram uma sequência mais longa e complexa de TCC (estilo Yang) por metade do tempo de intervenção (seis meses), constatando melhora no equilíbrio. Isso sugere que os efeitos do TCC sobre o equilíbrio podem estar mais ligados à complexidade da coreografia e à frequência semanal do que ao tempo da intervenção.

Nesta pesquisa, não houve diferenças significativas entre os grupos na flexibilidade, no equilíbrio estático, no alcance funcional e no risco de quedas, embora tenha sido observada a tendência do $\mathrm{G} 1$ a apresentar valores maiores do que o G2 nessas variáveis. Mesmo os autores que não encontraram evidências da eficácia do TCC na estabilidade postural, ${ }^{16}$ no equilíbrio, na RA e na força, ${ }^{17}$ reconhecem que há diminuição na incidência de quedas e no medo de cair nos idosos praticantes dessa modalidade de exercício.
Embora Logghe et al..$^{18}$ argumentem que a melhora da CF com a prática do TCC aplica-se somente aos idosos frágeis institucionalizados, nossos achados não são concordantes com essa afirmação. Nossa amostra foi constituída por idosos vivendo na comunidade, com alto nível de independência no desempenho das AVDs. Uma explicação para essa controvérsia é sugerida por $\mathrm{Li}$ et al., ${ }^{19}$ quando afirmam que carecem de maior rigor a qualidade das publicações sobre a prática do TCC em idosos, pois são poucos os estudos realizados com desenho e metodologia que permitam sua generalização e replicação dos resultados. Nesse sentido, a presente pesquisa aplicou rigor metodológico em todos os passos da intervenção, permitindo sua reprodução. Com a finalidade de diminuir viéses de seleção, neste estudo foram selecionados indivíduos que apresentaram medidas de base para idade, antropometria, DMO, CF e QV semelhantes o suficiente para que, estatisticamente, os dois grupos (experimental e controle) pudessem ser considerados equiparáveis. Uma característica frequente nas pesquisas com TCC em idosos consiste na utilização de grupos heterogêneos. Nesse sentido, este trabalho agrega valor à literatura ao controlar experimentalmente esses parâmetros.

A melhora em RA é um achado que levanta a necessidade de aprofundamento em futuras investigações sobre dose-resposta do TCC em indivíduos com baixa DMO, pois a intensidade do exercício prescrito na presente pesquisa não se encontrava na faixa de treinamento aeróbio para idosos em geral, mostrando-se suficiente para promover incrementos na RA dos homens portadores de baixa DMO.

$\mathrm{Na}$ presente pesquisa, o mesmo avaliador treinado para aplicação dos testes motores afasta, em grande parte, a possibilidade de viés de aferição. Cabe, no entanto, a possibilidade de que os resultados das medidas de desempenho físico tenham alguma influência de fatores motivacionais.

\section{Qualidade de vida}

$\mathrm{Na}$ literatura, somam-se evidências dos efeitos benéficos da prática do TCC sobre a $\mathrm{QV} .{ }^{9,20,21} \mathrm{O}$ estudo atual mostrou escore total da $\mathrm{QV}$ significativamente maior entre praticantes de TCC comparados ao G2. Jahnke et al., ${ }^{20} \mathrm{em}$ revisão, relataram que a maior parte dos estudos indica que o TCC tem grande potencial para incrementar a $\mathrm{QV}$, tanto em idosos saudáveis quanto naqueles portadores de doenças crônicas.

Os defensores dos efeitos do TCC sobre a QV, por sua vez, apresentam opiniões diversas sobre a abrangência desses efeitos. Relatos afirmam que o TCC melhora todas as dimensões da $\mathrm{QV} .{ }^{21}$ Entretanto, para alguns pesquisadores, idosos praticantes de TCC apresentam melhora principalmente nos domínios capacidade física e saúde da QV em relação ao G2, ${ }^{22}$ enquanto outros relatam que sua prática é capaz de incrementar ganhos 
em dimensões como vitalidade, física e social. ${ }^{23} \mathrm{~A}$ dimensão vitalidade, um dos aspectos do bem-estar físico, tem relação com balanço. A melhora no bem-estar físico pode também estar relacionada à melhoria dos distúrbios do sono noturno $\mathrm{e}$ à diminuição da sonolência diurna, resultando em melhora não farmacológica das alterações do sono em idosos. ${ }^{24}$ Outro possível mecanismo desse benefício está relacionado ao efeito da atividade física na função cerebrovascular.

São várias as possíveis causas dos efeitos positivos do TCC na QV.Idosos fisicamente ativos relatam melhor $\mathrm{QV}$ do que os sedentários. ${ }^{25}$ No estudo atual, não foi averiguado se os homens estudados eram sedentários no início da intervenção. Irwin et al. ${ }^{24}$ atribuem o efeito benéfico do TCC na QV ao relaxamento e exercício respiratório diafragmático acompanhado de meditação mental durante sua prática. Yau e Packer ${ }^{26}$ consideram que a expectativa dos participantes, que associam essa atividade com saúde e tranquilidade mental, faz com que melhorem por sugestionamento. Jin ${ }^{27}$ acrescenta que os praticantes dessa atividade esquecem os tópicos que lhes trazem ansiedade por consequência da concentração produzida durante o exercício. A importância da expectativa dos participantes e do efeito placebo ainda necessita de novas pesquisas.

Um determinante dos efeitos benéficos psicológicos do TCC consiste na personalidade dos praticantes e suas diferenças socioculturais. Toda et al. ${ }^{28}$ relatam que o TCC tem efeito significativamente mais benéfico nos domínios bem-estar emocional (depressão, humor, raiva e confusão) e bem-estar físico (fadiga) nos portadores de uma personalidade "impaciente" do que naqueles classificados como "calmos". Taylor-Piliae et al. ${ }^{29}$ apontam que um fator que pode levar a erro nos resultados é o estudo ser feito em grupo étnico asiático, que possui a tradição cultural de não mostrar emoções negativas em público e se conformar com as regras sociais.

\section{CONSIDERAÇÕES FINAIS}

O presente estudo apresentou limitações a serem sanadas em futuras intervenções. A primeira foi a baixa adesão ao protocolo, representada pela aceitação de somente 78 indivíduos para participar do estudo, em face dos 297 homens idosos cadastrados no programa de prevenção e diagnóstico na unidade de saúde.

A segunda, no nível estatístico, tem relação com o tamanho amostral: verificou-se que, para garantir baixa probabilidade de erro tipo II ( $\beta \leq 20 \%)$ e poder acima de $80 \%$, seriam necessários 28 indivíduos por grupo, o que não foi alcançado. Assim, nesta pesquisa, procurou-se controlar com maior rigor a probabilidade de erro tipo I $(\alpha \leq 5 \%)$.

Outra limitação encontrada foi a dos testes utilizados para avaliar a CF, todos submáximos e indiretos. Esses testes são validados e indicados como mais seguros para a população idosa, sendo escolhidos como primeira opção devido às características do grupo de idosos portadores de doença crônica. Entretanto, os valores iniciais elevados na maior parte deles indicam que futuros trabalhos deverão utilizar métodos mais diretos e sensíveis.

Uma investigação adicional deve ser a comparação entre diferentes estilos de TCC, assim como o nível de resposta dos indivíduos conforme as doenças de base que apresentem.

Os dados obtidos indicam que o TCC é eficaz em produzir melhora relativa da $\mathrm{CF}$ e da $\mathrm{QV}$ em homens idosos com baixa DMO. Os incrementos na $\mathrm{CF}$ relacionados à RA, FMMSS e FMMII, assim como no equilíbrio dinâmico, estendem para homens com baixa DMO a indicação clinicamente fundamentada da prática do TCC, já verificados em outros grupos de idosos de ambos os gêneros.

Assim, com os resultados observados no presente estudo, sugere-se o TCC como alternativa para treinamento físico seguro, a fim de incrementar a $\mathrm{CF}$ e a $\mathrm{QV}$ em homens portadores de baixa DMO. Justifica-se, ainda, a oferta de sua prática em programas visando ao atendimento de grandes populações, e, mais especificamente, em meio à rede pública de atendimento à saúde, em situações nas quais, do ponto de vista técnico ou econômico, não seja possível implantar programas de exercícios que necessitem de equipamentos, espaços ou vestimentas específicos.

\section{CONFLITO DE INTERESSES}

Os autores declaram não haver conflito de interesses.

\section{REFERÊNCIAS}

1. Martini LA, Moura EC, Santos LC, Malta DC, Pinheiro MM. Prevalência de diagnóstico auto-referido de osteoporose, Brasil, 2006. Rev Saúde Pública. 2009;43(Suppl. 2):107-16.

2. Lopes RF, Marchesi AO, Fossari RN, Cezar MC, Coeli CM, Farias MLF. Análise densitométrica da região femoral de homens acima de 50 anos oriundos de um ambulatório de urologia. Rev Bras Reumatol. 2009;49:402-12.
3. Compston J, Cooper A, Cooper C, Francis R, Kanis JA, Marsh D, et al. Guidelines for the diagnosis and management of osteoporosis in postmenopausal women and men from the age of 50 years in the UK. Maturitas. 2009;62:105-8.

4. Rolland $Y$, Kan GA, Bénétos A, Blain $H$, Bonnefoy $M$, Chassagne $P$, et al. Frailty, osteoporosis and hip fracture: causes, consequences and therapeutic perspectives. J Nutr Health Aging. 2008;12:335-46. 
5. Aldahr MHS. Bone mineral status response to aerobic versus resistance exercise training in postmenopausal women. World Appl Sci J. 2012;16:806-13.

6. Huang Y, Liu X. Improvement of balance control ability and flexibility in the elderly Tai Chi Chuan (TCC) practitioners: A systematic review and meta-analysis. Arch Gerontol Geriat. 2015;60:233-8.

7. Lan C, Chen SY, Lai JS, Wong AMK. Tai Chi Chuan in medicine and health promotion. Evid Based Complement Alternat Med. 2013. Article ID 502131:1-17.

8. Hall A, Maher C, Latimer J, Ferreira M. The effectiveness of tai chi for chronic musculoskeletal pain conditions: a systematic review and meta-analysis. Arthritis Rheum. 2009;61:717-24.

9. Wang C, Bannuru R, Ramel J, Kupelnick B, Scott T, Schmid CH. Tai Chi on psychological well-being: systematic review and meta-analysis. BMC Complement Alternat Med. 2010;10:23.

10. Brandão CMA, Camargos BM, Zerbini CA, Plapler PG, Mendonça LMC, Albergaria B, et al. Posições oficiais 2008 da Sociedade Brasileira de Densitometria Clínica (SBDens). Arq Br Endocrinol Metab. 2009;53:107-12.

11. Rikli RE, Jones CJ. Developmental and validation of a functional fitness test for community-residing older adults. J Aging Phys Act. 1999;7:129-61.

12. Rogers CR, Larkey LK, Keller C. A Review of Clinical Trials of Tai Chi and Qigong in Older Adults. West J Nurs Res. 2009;31:245-79.

13. Voukelatos A, Cumming RG, Lord SR, Rissel C. A randomized, controlled trial of tai chi for the prevention of falls: the Central Sydney tai chi trial. J Am Geriatr Soc. 2007;55:1185-91.

14. Li Y, Devault CN, Van Oteghen S. Effects of extended Tai Chi intervention on balance and selected motor functions of the elderly. Am J Chinese Med. 2007;35:383-91.

15. Yang Y, Verkuilen JV, Grubisich SA, Reed MR, Rosengren KS. The effect of Taiji (T'ai Chi/Qigong/ Ch'i Kung) on balance in older adults. Carle Selected Papers. 2007;50:8-18.

16. Marinho MS, Silva JF, Pereira LSM, Salmela LFT. Efeitos do Tai Chi Chuan na incidência de quedas, no medo de cair e no equilíbrio em idosos: uma revisão sistemática de ensaios clínicos aleatorizados. Rev Bras Geriat Gerontol. 2007;10:243-56.

17. Woo J, Hong A, Lau E, Lynn H. A randomized controlled trial of Tai Chi and resistance exercise on bone health, muscle strength and balance in community-living elderly people. Age Ageing. 2007;36:262-8.
18. Logghe IHJ, Verhagen AP, Rademaker ACHJ, Bierma-Zeinstra SMA Rossum E, Faber MJ, et al. The effects of Tai Chi on fall prevention, fear of falling and balance in older people: A meta-analysis. Prev Med. 2010;51:222-7.

19. Li J, Zhang YF, Smith GS, Xue CJ, Luo YN, Chen WH, et al. Quality of Reporting of Randomized Clinical Trials in Tai Chi Interventions - A Systematic Review. Evid Based Complement Alternat Med. 2011;2011:1-10.

20. Jahnke R, Larkey L, Rogers C, Etnier J, Lin F. A comprehensive review of health benefits of qigong and tai chi. Am J Health Promot. 2010;24:e1-25

21. Lee LYK, Lee DTF, Woo J. Effect of tai chi on state self-esteem and health-related quality of life in older Chinese residential care home residents. J Clin Nurs. 2007;16:1580-2

22. Ho TJ, Liang W-M, Lien CH, Ma TC, Kuo HW, Chu BC, et al. Healthrelated quality of life in the elderly practicing Tai Chi Chuan. J Alternat Complement Med. 2007;13:1077-84.

23. Tsang T, Rhonda O, Lam P, Comino EJ, Fiatarone-Singh M. Health benefits of tai chi for older patients with type 2 diabetes: the "move it for diabetes study" - A randomized controlled trial. Clin Interv Aging. 2007;2:429-39.

24. Irwin MR, Olmstead R, Motivala SJ. Improving sleep quality in older adults with moderate sleep complaints: a randomized controlled trial of Tai Chi Chuan. Sleep. 2008;31:1001-8.

25. Oliveira AC, Oliveira NMD, Arantes PMM, Alencar MA. Qualidade de vida em idosos que praticam atividade física - uma revisão sistemática. Rev Bras Geriat Gerontol. 2010;13:301-12

26. Yau MK, Packer TL. Health and well-being through Tai Chi: perceptions of older adults in Hong Kong. Leisure Stud. 2002;21:163-78.

27. Jin P. Changes in heart rate, noradrenaline, cortisol and mood during Tai Chi. J Psychosom Res. 1989;33:197-206.

28. Toda M, Den R, Hasegawa-Ohira M, Morimoto K. Influence of personal patterns of behavior on the effect of Tai Chi: a pilot study. Environ Health Prev Med. 2011;16:61-3.

29. Taylor-Piliae RE, Haskell WL, Waters CM, Froelicher ES. Change in perceived psychosocial status following a 12-week Tai Chi exercise program. J Adv Nurs. 2006;54:313-29. 